\title{
A Comparison of two Methods for Fusing Information from a Linear Array of Sonar Sensors for Obstacle Localization *
}

\author{
Orhan Arlkan and Billur Barshan \\ Department of Electrical Engineering \\ Bilkent University \\ Bilkent, 06533 Ankara, Turkey
}

\begin{abstract}
The performance of a commonly employed linear array of sonar sensors is assessed for point-obstacle localization intended for robotics applications. Two different methods of combining time-of-fight information from the sensors are described to estimate the range and azimuth of the obstacle: pairwise estimate method and the maximum likelihood estimator. The variances of the methods are compared to the Cramér-Rao Lower Bound, and their biases are investigated. Simulation studies indicate that in estimating range, both methods perform comparably; in estimating azimuth, maximum likelihood estimate is superior at a cost of extra computation. The results are useful for target localization in mobile robotics.
\end{abstract}

\section{Introduction}

In this paper, the performance of a commonly employed linear array of sonar sensors is assessed for point-target localization. Characterizing point-target response of a sensor has been important not only for its application to point targets but also to assess its performance on extended targets which can be modeled using different approaches $[1,2,3]$. If the approach is one of hypothesis testing or one of parametrizing the extended target, then sensor performance may not be easily related to its point-target response. On the other hand, for extended targets of unknown shape with possible roughness [4], or for small spherical targets, point target analysis can be extremely useful. Aside from modelling extended targets, the point target analysis can be easily extended to spherical targets of finite radius which may be of interest in robotics applications. In this study, only point targets are considered. By implementing a multi-transducer system that exploits the differences in the signal travel times and combining information from the array elements, the location of a point target can be accurately estimated in two dimensions.

In the next section, the transducer model and the linear array configuration are described. In Section 3, two different approaches for point target localization are described and the Cramér Rao Lower Bound (CRLB) is derived. The performances of the

*This work was supported by TUBITAK EEEAG-92 project.

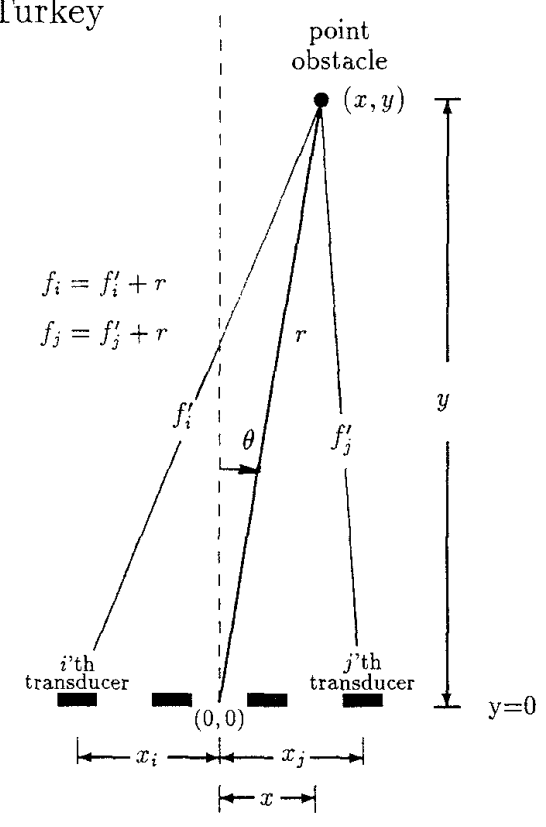

Figure 1: A linear array of $N=4$ transducers for localization.

two methods are discussed in terms of bias and variance, and their variances are compared to the CRLB in Section 4. In the concluding section, the usefulness of the methods is assessed for point-target localization.

\section{The Sensor Model and the Array Configuration}

A single acoustic transducer can be employed both as a transmitter and receiver. After the transmitted pulse encounters an object, an echo is detected by the same transducer acting as a receiver. In this study, the excitation is chosen to be a gated Gaussian-modulated sinusoid. Localization of a point target is performed in the far-zone of the transducer where the propagating pulse is considered to be a series of plane waves.

In this investigation, it is assumed that the point target and all the transducers lie in the same plane as illustrated in Figure 1 for $N=4$ transducers. Uniformly spaced sensors in this array are modeled as 
identical Polaroid sensors pointing in the same direction. Both of these assumptions can be relaxed without significant change in the following development.

Suppose there is a point obstacle located at $(x, y)$ and that the $i$ 'th transducer transmits a pulse whose mathematical model will be provided in the next section. The rectangular coordinates of the $i$ th and $j$ 'th transducers, where $i, j=1, \ldots, N$, are $\left(x_{i}, 0\right)$ and $\left(x_{j}, 0\right)$ respectively as shown in Figure 1 for $N=4$. The distance-of-flight (DOF) measured at transducer $i$ is $f_{i}$, and the corresponding DOF at $j$ is $f_{j}$. These DOF's at the two transducers define a circle and an ellipse whose point of intersection with a positive $y$ coordinate corresponds to the obstacle location. Solving for the intersection point:

$$
\begin{aligned}
x_{1,2} & =\frac{2 f_{j}\left(f_{j} \pm f_{i}\right)}{x_{i}-x_{j}}+\frac{x_{i}+x_{j}}{2} \\
y & =\sqrt{f_{i}^{2}-\left(x-x_{i}\right)^{2}}
\end{aligned}
$$

One of the two roots $x_{1,2}$ is chosen as $x$ such that $f_{i}^{2}-\left(x-x_{i}\right)^{2}$ is positive. Using $x$ and $y$, the polar coordinates of the target can be found as

$$
\begin{aligned}
& r=\sqrt{x^{2}+y^{2}} \\
& \theta=\sin ^{-1}\left(\frac{x}{r}\right)
\end{aligned}
$$

\section{Estimation of Point-Target Position}

\subsection{Description of the two Methods}

Using the given array configuration, information from the sensors can be combined in a number of ways. In earlier work, finding the optimal receiver separation at a given range for plane-corner differentiation was considered and the pair that best approximates this separation in a linear array of $N$ transducers was chosen [5]. With the same configuration, fusing information pairwise from all pairs of receivers symmetric around the center of the array have been investigated and the 'optimal' weighting factors for the estimates from these pairs were found [5]. This method improved the accuracy of the estimates approximately by $10 \%$ although the processing time was increased threefold for $N=6$. This method will be referred as the $s u b$-array method since it does not make use of all the received signals available in the system.

In the array configuration assumed here, every transducer takes turn in transmitting, and after each transmission, received waveforms are recorded at every transducer. Hence, after a full cycle of transmission, there are $N^{2}$ received waveforms. This allows us to extend the sub-array method to a more complete one in which every available echo is used. In total, there are $N(N-1)$ such pairs from which both $\theta$ and $r$ estimates can be obtained [5]. This method will be referred as the pairwise estimate (PE) method. Although this extension makes more complete use of the acquired data in localizing the point obstacle, a single, robust location estimate needs to be extracted from the data. From the geometry of Figure 1, $r$ and $\theta$ estimates (given by Equation 3) are obtained at each receiver when one of the $N$ transducers is used as a transmitter. These $N(N-1)$ estimates are combined by calculating their mean and excluding any estimate not within two standard deviations of the mean while doing so.

In a second approach, all received waveforms are considered at the same time and the best $r$ and $\theta$ which provide the most probable fit (the MLE) to the acquired data are chosen as the final estimate. This procedure requires the use of nonlinear iterative optimization techniques. Since the cost function used in this optimization procedure is observed to have multiple local minima, the choice of the starting point is important in reaching the optimal values. One good choice is the minimum of the cost function on a coarse mesh centered around the PE result. The minimum so obtained is used as an initial estimate to find an approximation to the MLE of $r$ and $\theta$ by minimizing the cost function described below.

The following additive noise signal-observation model is assumed:

$$
r_{i j}\left(t_{k}, \mathbf{z}\right)=s_{i j}\left(t_{k}, \mathbf{z}\right)+n_{i j}\left(t_{k}\right)
$$

for $i, j=1, \ldots, N$ and $k=1, \ldots, M$. Here, $r_{i j}\left(t_{k}\right)$ is the received waveform at time sample $t_{k}$ at the $j$ 'th transducer when the $i$ 'th transducer is activated. The vector $\mathbf{z}$ is the location parameter vector of the pointtarget given by

$$
\mathbf{z}=\left[\begin{array}{l}
r \\
\theta
\end{array}\right]
$$

$s_{i j}\left(t_{k}, r, \theta\right)$ is modeled as follows:

$s_{i j}\left(t_{k}, r, \theta\right)=A\left(x_{i}, r, \theta\right) A\left(x_{j}, r, \theta\right) G\left(x_{i}, r, \theta\right) G\left(x_{j}, r(\theta)\right.$

$M\left[t_{k}-t_{i}(r, \theta)-t_{j}(r, \theta)\right] \cos \left\{2 \pi f_{\circ}\left[t_{k}-t_{i}(r, \theta)-t_{j}(r, \theta)\right]\right\}(7)$

where

$$
\begin{aligned}
A\left(x_{i}, r, \theta\right) & =\frac{1}{2 \sqrt{\pi\left[r^{2} \cos ^{2} \theta+\left(r \sin \theta-x_{i}\right)^{2}\right]}}(8) \\
G\left(x_{i}, r, \theta\right) & =\frac{2 J_{1}\left[k a \frac{\left(r \sin \theta-x_{i}\right)}{\sqrt{r^{2} \cos ^{2} \theta+\left(r \sin \theta-x_{i}\right)^{2}}}\right]}{k a \frac{\left(r \sin \theta-x_{i}\right)}{\sqrt{r^{2} \cos ^{2} \theta+\left(r \sin \theta-x_{i}\right)^{2}}}}(9) \\
M[t] & =\exp \left(\frac{-t^{2}}{2 \sigma^{2}}\right) \operatorname{rect}\left(\frac{f_{0} t}{5}\right) \\
t_{i}(r, \theta) & =\frac{\sqrt{r^{2} \cos ^{2} \theta+\left(r \sin \theta-x_{i}\right)^{2}}}{c}
\end{aligned}
$$

where $k=\frac{2 \pi}{\lambda}, c$ is the speed of sound in air, and $f_{o}=50 \mathrm{kHz}$ is the resonant frequency of the Polaroid transducer with aperture radius $a=2 \mathrm{~cm}$. Here, $A\left(x_{i}, r, \theta\right)$ is the free-space attenuation factor of the pressure amplitude, $G\left(x_{i}, r, \theta\right)$ is the gain pattern of the transducer, and $M[t]$ is the envelope of the waveform modeled as a gated Gaussian. 
Note that the received waveform has nonlinear dependence on range and azimuth. It is desirable to have unbiased $r$ and $\theta$ estimates based on the acquired array data. In this type of nonlinear estimation problems, it is difficult to find an exact expression for the variance of the estimate. In the following section, the performance of any nonlinear unbiased estimator will be characterized by deriving a lower bound on its variance.

\subsection{Derivation of the Cramér-Rao Lower Bounds}

Cramér-Rao Lower Bound (CRLB) defines a lower bound on the variance of any unbiased estimator [6]. To find the CRLB in this particular case, an independent identically distributed Gaussian noise model is assumed with the following conditional probability density function:

$p_{\mathbf{r} \mid \mathbf{Z}}(\mathbf{r} \mid \mathbf{z})=\prod_{i=1}^{N} \prod_{j=1}^{N} \prod_{k=1}^{M} \frac{1}{\sqrt{2 \pi \sigma^{2}}} \exp -\frac{\left[r_{i j}\left(t_{k}\right)-s_{i j}\left(t_{k}, \mathbf{z}\right)\right]^{2}}{2 \sigma^{2}}$

The MLE estimate given above chooses that value of $\mathbf{z}$ which maximizes the conditional probability. By taking the natural logarithm of both sides, we obtain a simpler expression to be maximized:

$\ln p_{\mathbf{r} \mid \mathbf{Z}}(\mathbf{r} \mid \mathbf{z})=\sum_{i, j, k}\left\{\ln \left(\frac{1}{\sqrt{2 \pi \sigma^{2}}}\right)-\frac{\left[r_{i j}\left(t_{k}\right)-s_{i j}\left(t_{k}, \mathbf{z}\right)\right]^{2}}{2 \sigma^{2}}\right\}$

From above, the final form of the cost function to be minimized for the MLE is

$$
\sum_{i, j, k} \frac{\left[r_{i j}\left(t_{k}\right)-s_{i j}\left(t_{k}, \mathbf{z}\right)\right]^{2}}{2 \sigma^{2}}
$$

Due to the nonlinearity of the expression in $z$, an exact expression for the MLE is difficult to find, and an iterative numeric method is used. From the above expression the CRLB can be derived by computing the following partial derivatives of Equation 13

$$
\begin{aligned}
-\frac{\partial \ln p_{\mathbf{r} \mid \mathbf{Z}}(\mathbf{r} \mid \mathbf{z})}{\partial z_{n}}= & -\sum_{i, j, k} \frac{\left[r_{i j}\left(t_{k}\right)-s_{i j}\left(t_{k}, \mathbf{z}\right)\right]}{\sigma^{2}} \frac{\partial s_{i j}\left(t_{k}, \mathbf{z}\right)}{\partial z_{n}} \\
-\frac{\partial^{2} \ln p_{\mathbf{r} \mid \mathbf{Z}}(\mathbf{r} \mid \mathbf{z})}{\partial z_{n} \partial z_{m}}= & -\sum_{i, j, k} \frac{\left[r_{i j}\left(t_{k}\right)-s_{i j}\left(t_{k}, \mathbf{z}\right)\right]}{\sigma^{2}} \frac{\partial^{2} s_{i j}\left(t_{k}, \mathbf{z}\right)}{\partial z_{n} \partial z_{m}} \\
& +\frac{1}{\sigma^{2}} \sum_{i, j, k} \frac{\partial s_{i j}\left(t_{k}, \mathbf{z}\right)}{\partial z_{n}} \frac{\partial s_{i j}\left(t_{k}, \mathbf{z}\right)}{\partial z_{m}}
\end{aligned}
$$

where the righthandside for $n, m=1,2$ defines the entries of $\mathbf{J}$, the Fisher Information Matrix [6]. Then the expected value of $\mathbf{J}$ is:

$$
E\{\mathbf{J}\}=\mathbf{H}
$$

where

$$
H(n, m)=\frac{1}{\sigma^{2}} \sum_{i, j, k} \frac{\partial s_{i j}\left(t_{k}, \mathbf{z}\right)}{\partial z_{n}} \frac{\partial s_{i j}\left(t_{k}, \mathbf{z}\right)}{\partial z_{m}} \quad n, m=1,2
$$

Then, the CRLB becomes

$$
E\left\{\left(\hat{z}_{n}-z_{n}\right)^{2}\right\} \geq \frac{1}{H^{-1}(n, n)}
$$

Here, $\hat{z}$ is any unbiased estimate of the parameter vector elements $r$ and $\theta$.

To find the expressions in Equation 15, partial derivatives of the amplitude and gain terms in Equation 8 were evaluated.

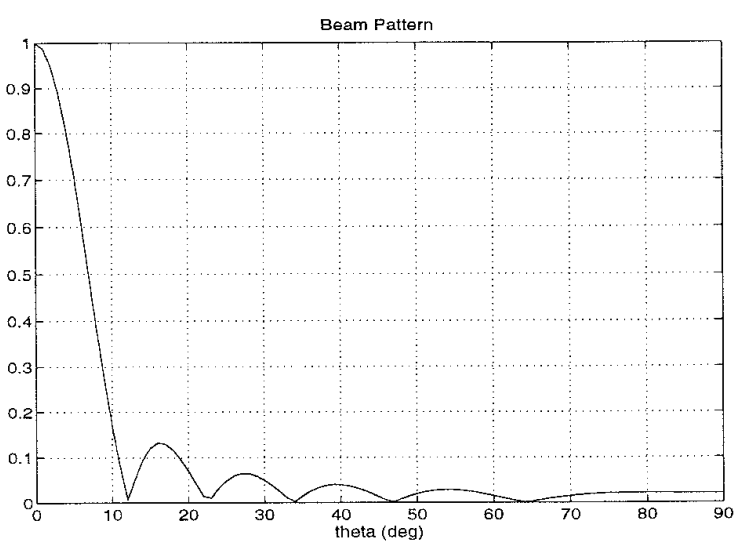

Figure 2: Transducer beam pattern at the resonant frequency $f_{0}=50 \mathrm{kHz}$.

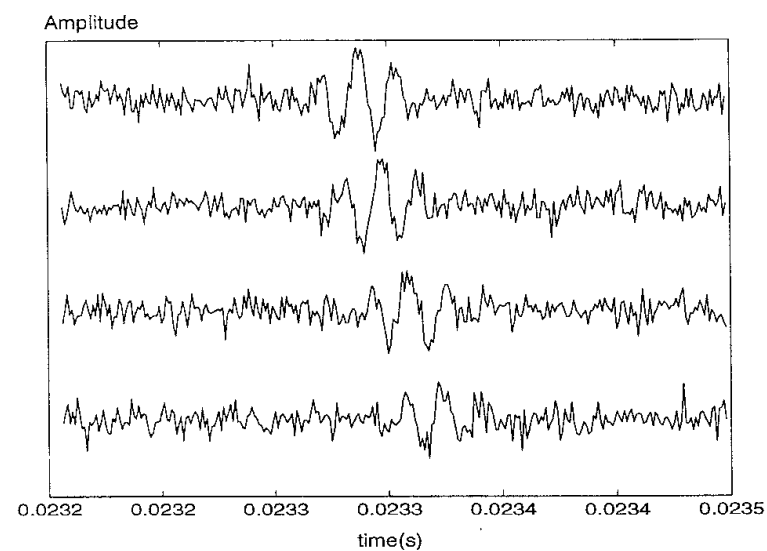

Figure 3: Received waveforms at each transducer when $r=4 \mathrm{~m}$.

In the next section, performances of both of the PE and MLE for point-obstacle localization will be investigated and compared to the CRLB over some synthetic test cases. 


\section{Results and Discussion}

The results presented in this section are obtained from a simulation study for an array of $N=4$ transducers of Polaroid sensors with resonant frequency $f_{0}=50 \mathrm{kHz}$ and separation $6 \mathrm{~cm}$. Neglecting the narrow bandwidth around $f_{0}$, the beam pattern of the transducer is a first-order Bessel function of the first kind as illustrated in Figure 2.

Figure 3 displays received waveforms at each transducer when the leftmost one transmits. The point obstacle is located at a range of $r=4 \mathrm{~m}$ and an azimuth of $\theta=4^{\circ}$. The standard deviation of the added Gaussian noise is chosen to be $5 \%$ of the maximum signal amplitude at a range of $1 \mathrm{~m}$.

In this simulation study, statistical performances of the two methods (PE and MLE) are compared with each other as well as with the CRLB derived previously. Each of the $N(N-1)$ pairs provides a single range and azimuth estimate. The mean values and standard deviations of these estimates are computed based on ten different realizations for a given range and azimuth. Figure 4, shows the standard error of the range and azimuth estimates that are obtained from $\mathrm{PE}$ when the azimuth is kept constant at $\theta=0^{\circ}$, and the range $r$ is changed between $1-4 \mathrm{~m}$ with $25 \mathrm{~cm}$ increments. The standard error is found by summing the squares of standard deviation and the bias of the estimate and then taking the square root. The corresponding results for the MLE are given in Figure 5 . Both of these approaches indicate similar trends. The standard deviation of MLE in $r$ is slightly more than that of PE. The slight increase in the standard deviations as $r$ increases is due to the decrease in the signal-to-noise ratio because of the free-space attenuation factor. For comparison purposes, the CRLB for unbiased estimators has also been included in each figure. It is not possible to make a comparison to the CRLB for biased estimators since an analytic expression for the bias is not available. The estimation bias partially derives from that one already present in the raw DOF measurements since these measurements are obtained by thresholding [7]. Although these standard deviations have an increasing trend as a function of $r$, even at $r=4 \mathrm{~m}$, their values are of the order of $1 \mathrm{~cm}$ in $r$ and approximately $0.1^{\circ}$ in $\theta$. These values are acceptable in practice.

The results obtained when $r$ is kept constant at 2 $\mathrm{m}$ and $\theta$ is varied between $0-4^{\circ}$ with increments of $1^{\circ}$ is illustrated in Figures 8 and 9 . All of the above observations apply to these two cases as well. In this case, the increasing trend with $\theta$ is due to the reduced transducer gain as a function of increasing $|\theta|$.

Biases of these estimates have also been investigated based on the same set of simulations. The results are shown in Figures 6,7,10 and 11. In the first two figures, biases of $\mathrm{PE}$ and $\mathrm{MLE}$ as function of $r$ have been displayed when $\theta$ is kept constant at $0^{\circ}$. For a better display, the data points have been fitted with a spline. The bias in estimating range as a function of $r$ in both cases is acceptable, at worst 5.9 $\mathrm{mm}$. Corresponding biases in the azimuth estimates are also within the acceptable range of $\pm 0.14^{\circ}$ for $\mathrm{PE}$ and $\pm 0.055^{\circ}$ for MLE. In the last two figures, biases as functions of $\theta$ are investigated at a constant range of $r=2 \mathrm{~m}$. Again, the available data has been interpolated by a spline fit. All of these biases are also within acceptable levels.

Based on this simulation study, it has been observed that although in estimating range, both PE and MLE perform comparably, MLE is superior in estimating the azimuth of the point target.

\section{Conclusion}

Two different methods of fusing information from a linear array of $N$ acoustic transducers for estimating the position of a point target have been described. The methods are characterized by small biases, and standard errors larger than the CRLB by an order of 2-4. Although the PE and MLE methods provide similar range estimation accuracy, MLE outperforms the PE in estimating azimuth.

This study is useful for characterizing the pointtarget response of acoustic sensors and forms a basis to find their response to rough surfaces which can be modeled as a random collection of point targets. The system is being implemented in hardware and the obtained experimental results will be compared with the analytical and simulation results. The results are useful for target localization in mobile robotics.

\section{References}

[1] H. Peremans, K. Audenaert and J. M. Van Campenhout "A high-resolution sensor based on triaural perception," IEEE Transactions on Robotics and Automation, vol. 9, pp. 36-48, February 1993.

[2] J. J. Leonard and H. F. Durrant-Whyte "Mobile robot localization by tracking geometric beacons," IEEE Transactions on Robotics and Automation, vol. 7 , pp. $376-382,1991$.

[3] M. L. Hong and L. Kleeman "Analysis of ultrasonic differentiation of three-dimensional corners, edges and planes," in Proceedings IEEE International Conference on Robotics and Automation, pp. 580-584, Nice, France, May 12-14, 1992.

[4] Ö. Bozma and R. Kuc "Characterizing pulses reflected from rough surfaces using ultrasound," Journal of the Acoustical Society of America, vol. 89 , pp. $2519-2531$, June 1991.

[5] B. Barshan and R. Kuc "Differentiating sonar reflections from corners and planes by employing an intelligent sensor," IEEE Transactions on Pattern Analysis and Machine Intelligence, vol. 12, pp. 560-569, June 1990 .

[6] H. L. Van Trees. Detection, Estimation, and Modulation Theory, Part I. John Wiley \& Sons, New York, 1968.

[7] B. Barshan. A Sonar-Based Mobile Robot for BatLike Prey Capture. PhD thesis, Yale University, New Haven, CT, December 1991. University of Michigan Microfilms, order number 9224325 . 


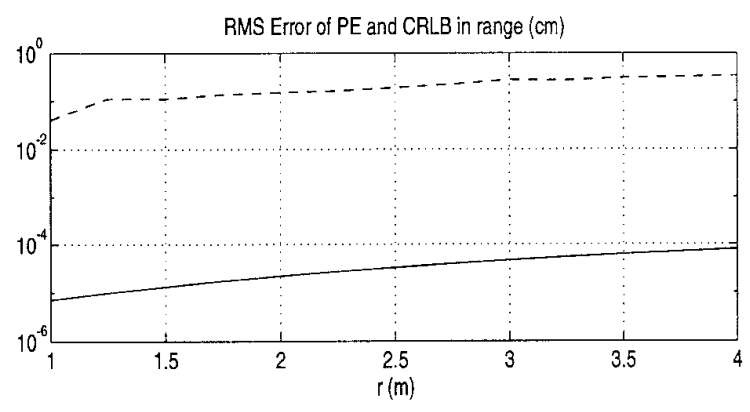

RMS Error of PE and CRLB in azimuth (deg)

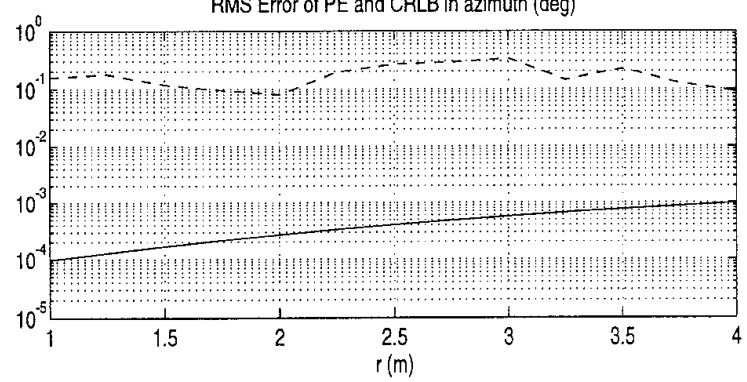

Figure 4: RMS error of $\mathrm{PE}$ in range and azimuth as a function of range when $\theta=0^{\circ}$ in dashed line. CRLB in solid line.

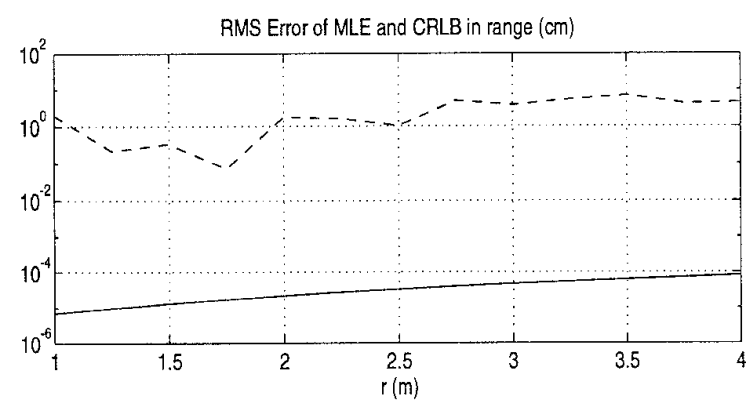

RMS Error of MLE and CRLB in azimuth (deg)

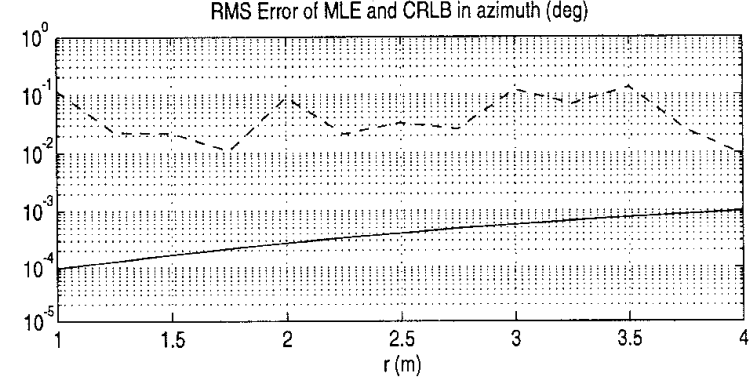

Figure 5: RMS error of MLE in range and azimuth as a function of range when $\theta=0^{\circ}$ in dashed line. CRLB in solid line.
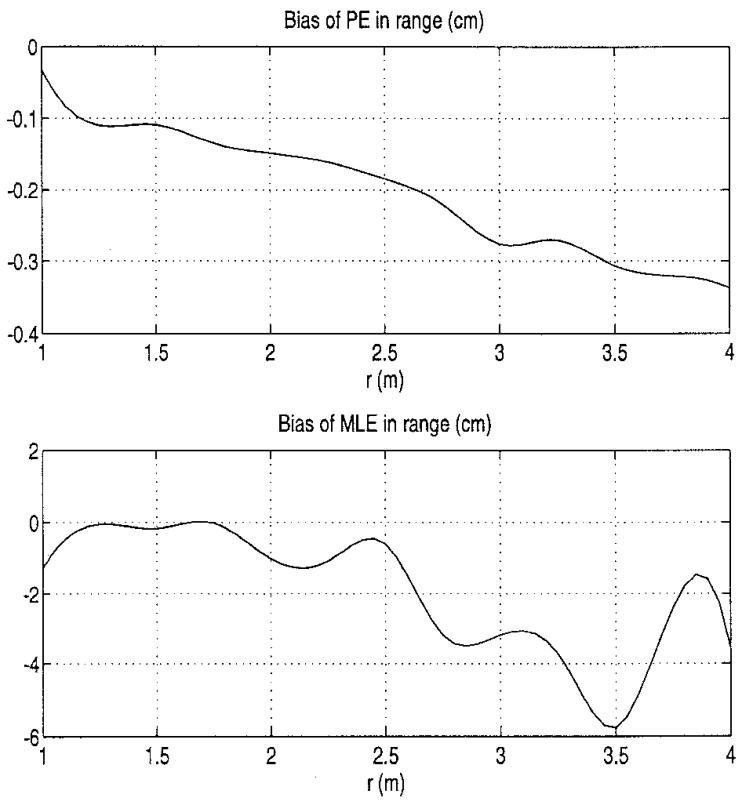

Figure 6: Bias of PE and MLE in range as a function of range when $\theta=0^{\circ}$.
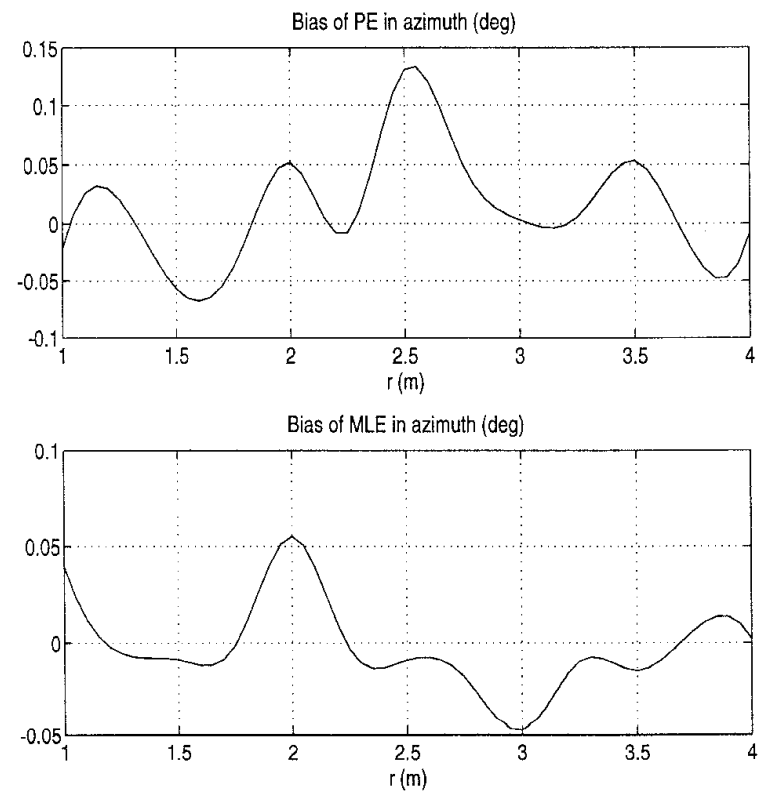

Figure 7: Bias of PE and MLE in azimuth as a function of range when $\theta=0^{\circ}$. 

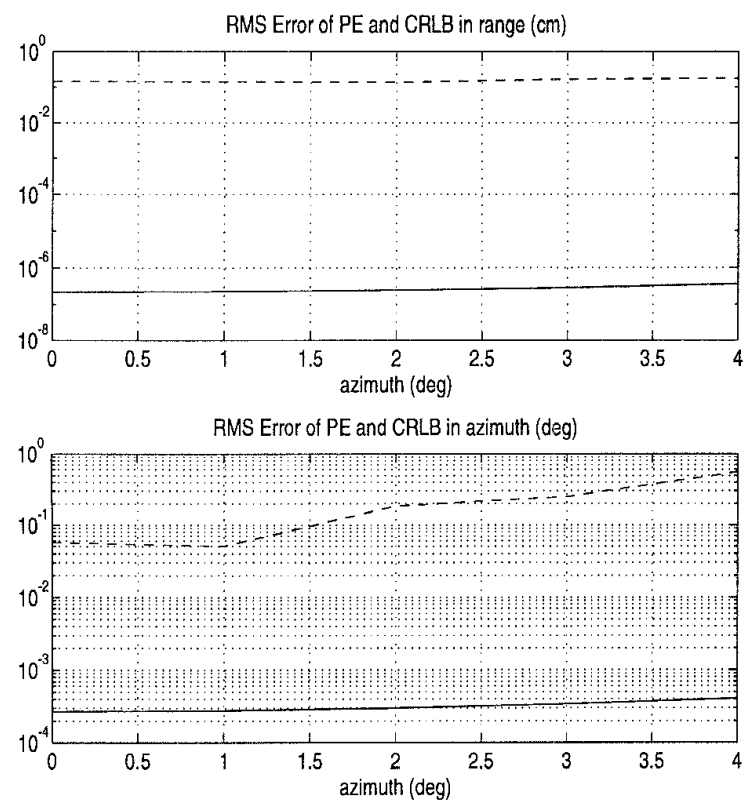

Figure 8: RMS error of $\mathrm{PE}$ in range and azimuth as a function of azimuth when $r=2 \mathrm{~m}$ in dashed line. CRLB in solid line.
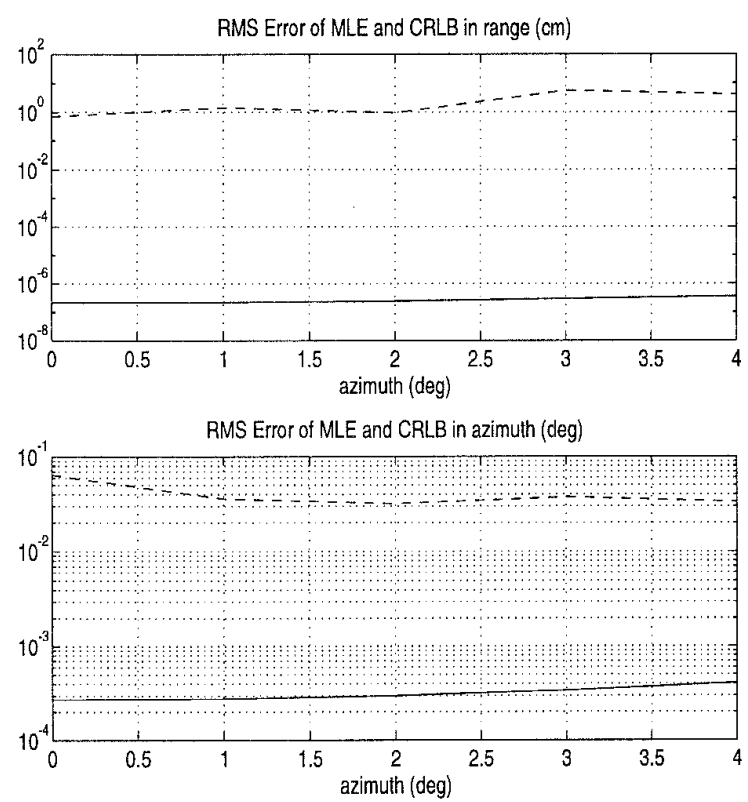

Figure 9: RMS error of MLE in range and azimuth as a function of azimuth when $r=2 \mathrm{~m}$ in dashed line. CRLB in solid line.
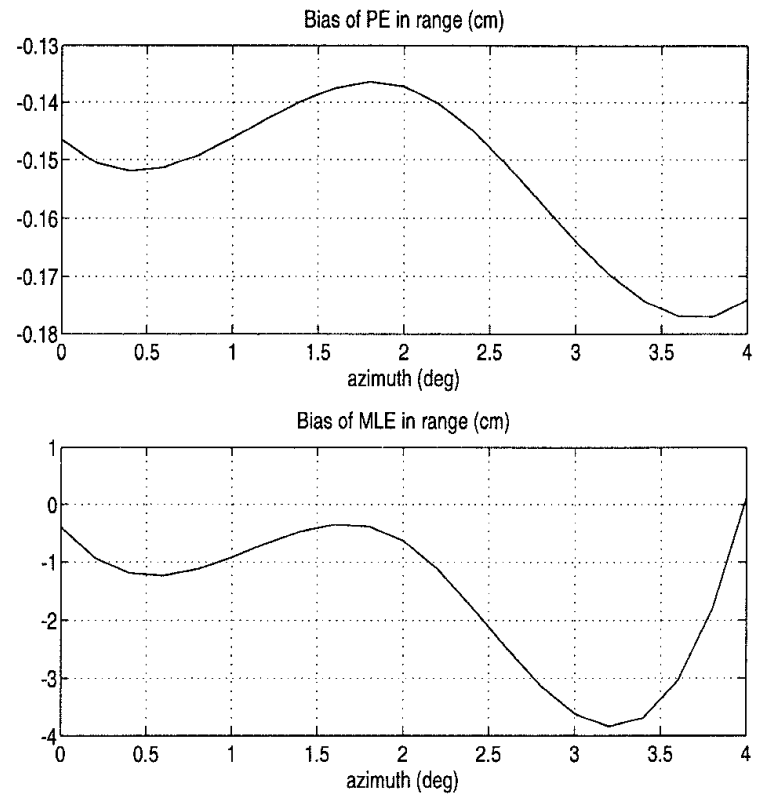

Figure 10: Bias of $\mathrm{PE}$ and MLE in range as a function of azimuth when $r=2 \mathrm{~m}$.
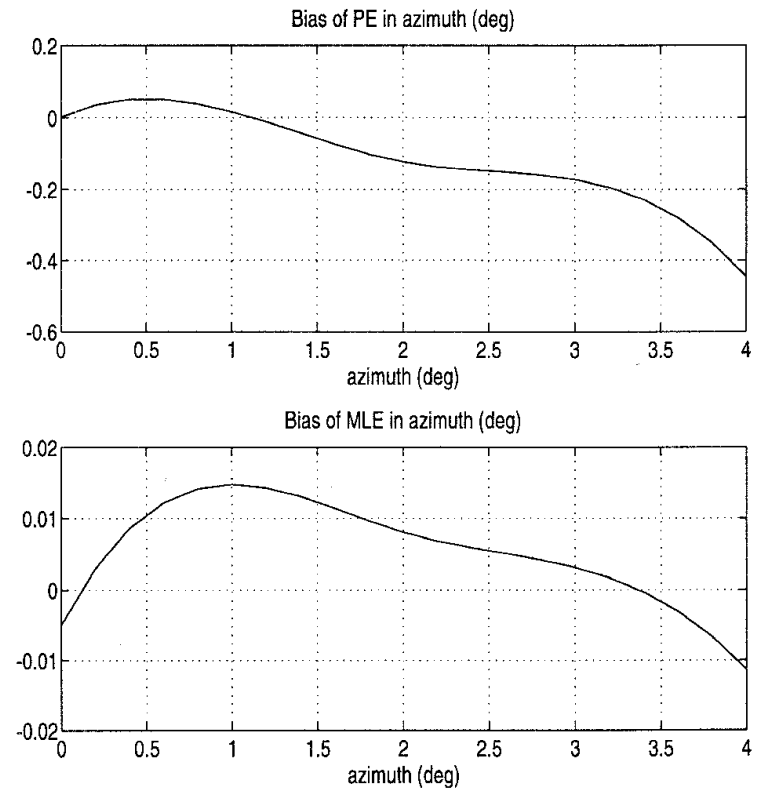

Figure 11: Bias of PE and MLE in azimuth as a function of azimuth when $r=2 \mathrm{~m}$. 